\title{
eGK: Per Knopfdruck ändern sich die Kartendaten
}

\section{Auch ohne Online-Phase lassen sich die Versichertendaten auf der elektro- nischen Gesundheitskarte bereits per Knopfdruck korrigieren.}

$\mathrm{M}$ it der elektronischen Gesundheitskarte (eGK) sollte Patienten eigentlich die Möglichkeit eingeräumt werden, ihre Versichertenstammdaten und diverse andere Gesundheitsdaten einsehen zu können. Zwar ist dieses Projekt samt Online-Phase etwas nach hinten verschoben worden, Anbieter, die hierfür für Patienten, Praxen und Krankenkassen funktionsfähige EDV-Systeme haben, gibt es aber bereits, wie die Computermesse CeBIT 2012 in Hannover zeigte. Eine solche Lösung im Gepäck hatte die Concat AG. Das System des E-Health-Anbieters besteht aus zwei Komponenten: einem System für die Krankenkassen und einem „Wartezimmer-Kiosk“.

Macht die Krankenkasse eines Patienten mit und nutzt die Anwendungsplattform, über die sie schon heute in jeder Geschäftsstelle die Funktionsfähigkeit der eGK prüfen kann, hat das auch für Versicherte Vorteile. Die können sich nämlich zu Hause über Web-Browser und mit eGK in die Anwendungssoftware einloggen und ihre Versicherungsgrunddaten einsehen. Michael Brockt von Concat zeigte auf der Messe, wie es funktioniert: Ist der Versicherte auf der Plattform eingeloggt, kann er mit einem gängigen USB-Kartenterminal die Daten seiner eGK aufrufen. Erkennt er einen Fehler in den Daten, korrigiert er diesen. Die neuen Daten werden zunächst im System gespeichert, noch nicht auf der eGK, wie Brockt erklärte. Denn noch läuft der Online-Abgleich der Versichertenstammdaten (VSD) ja nicht. Der Versicherte druckt sich den geänderten Datensatz daher aus und geht damit und mit seiner eGK in die Geschäftsstelle der Krankenkasse. Dort wird die Karte eingelesen und einfach per Knopfdruck ein „Update“ durchgeführt. Damit werden die neuen Daten auf der eGK gespeichert.

Auch auf dem Wartezimmer-Kiosk können sich Versicherte ihre Daten anzeigen lassen. Der Vorteil des Kiosks liegt laut Brockt aber an anderer Stelle: Der Kiosk könne bereits mit dem Konnektor ausgeliefert werden, dadurch könne beim späteren Online-Betrieb der eGK die Praxis-EDV weiterhin offline laufen. Denn die Daten der eGK ließen sich in einem gesicherten System-Container ablegen, für den nur die Praxis-EDV eine Leseberechtigung habe, um die Daten dann in die Patientenakten zu übernehmen. Der Versichertenstammdatenabgleich mit der Krankenkasse laufe dadurch getrennt von der Praxis-EDV nur über den Konnektor ab. Und an diesen ließen sich auch weitere Lesegeräte koppeln. Kostenmäßig soll der Kiosk - allerdings ohne Konnektor, aber mit Touchscreen und versiegeltem Kartenleser - unter $4.000 €$ kosten.

RebekkaHöhl
Windows 8 kommt mit Touchscreen-Oberfläche

Auf der CeBIT 2012 hat Microsoft die Betaversion "Consumer Preview" von Windows 8 vorgestellt. Und die ist selbst für geübte Windows-Nutzer etwas komplett Neues. Das System wurde nämlich konsequent an der Touch-Bedienung ausgerichtet. Das heißt, das bekannte Windows-Startmenü musste dem "Metro" genannten Startbildschirm weichen, auf dem Rechtecke für Inhalte und Programme stehen. Die einzelnen Programme und Inhalte lassen sich aber auch mithilfe der PC-Maus aufrufen falls Nutzer nicht über einen Touchscreen verfügen. Ebenfalls in die Metro-Oberfläche eingebunden wurde der neue Skydrive-Cloud-Speicher, über den jeder Nutzer kostenlos 25 GB Speicherplatz in der Cloud erhält. Rebekka Höhl

\section{Mobiles Internet begeistert Digital-Laien}

Tablet-Computer und Smartphones sprechen laut einer aktuellen Studie des Marktforschungsunternehmens TNS Infratest zunehmend digital unerfahrene Nutzer an - und tragen so dazu bei, die digitale Kluft zu schließen. Derzeit greifen der Studie zufolge bereits $26,5 \%$ der Bevölkerung in Deutschland von unterwegs auf das Internet zu. Für die Studie hatte TNS Infratest im Auftrag der Initiative D21, einem Netzwerk von Vertretern aus Wirtschaft und Politik, insgesamt 1.005 Telefoninterviews durchgeführt. Wenn das Mobilfunknetz leistungsfähiger wäre und Daten schneller übertragen würden, würden sogar $40 \%$ der Befragten deutlich häufiger mobil ins Netz gehen. 43,4\% der Befragten könnten sich sogar vorstellen, dann nur noch einen mobilen Zugang zu nutzen.

\section{Neues iPad 3 im Handel}

Das neue iPad 3 erobert derzeit den Markt. Es ist ein wenig dicker und schwerer als sein Vorgänger. Die wichtigste Neuerung beim neuen Apple Tablet PC ist das hochauflösende Display. Während der Vorgänger 1.024 mal 768 Pixel darstellte, kommt das iPad 3 auf 1.536 mal 2.048 Pixel, was eine Vervierfachung bedeutet. Insgesamt stellt der Bildschirm also 3,1 Megapixel dar, Apple nennt das Retina-Display. Bemerkenswert ist auch die Möglichkeit zur Rundumvernetzung - auch wenn das neue iPad mit den in Deutschland üblichen Frequenzen für den Mobilfunkturbo LTE noch nichts anfangen kann. 